\title{
Kinematics and Principal Component Analysis from IFU Spectroscopy of the Post-Starburst Quasar SDSS J0210-0903
}

\author{
David Sanmartim ${ }^{1}$, Thaisa Storchi-Bergmann ${ }^{1}$, and \\ Michael S. Brotherton ${ }^{2}$ \\ ${ }^{1}$ Instituto de Física, Universidade Federal do Rio Grande do Sul, RS, Brasil \\ Email: davidsanm@gmail.com, thaisa@ufrgs.br \\ ${ }^{2}$ Department of Physics and Astronomy, University of Wyoming, Laramie, WY 82071, USA \\ Email: mbrother@uwyo.edu
}

Keywords. galaxies: starburst, galaxies: quasar, galaxies: kinematics

We present 2-D mapping and analysis of the gaseous kinematics of the inner $7^{\prime \prime} \times 5^{\prime \prime}$ of one of nearest $(z=0.0414)$ and brightest post-starburst quasars (PSQ) by using spectra obtained with the Integral Field Unit (IFU) of the Gemini Multi-Object Spectrograph on the Gemini North Telescope (Allington-Smith et al. 2002). Such quasars are broad-lined AGNs that also show the Balmer jumps and the high-order Balmer absorption lines from A stars typical of massive poststarburst populations of a few hundred Myrs (Brotherton et al. 2007). From measurements of the emission-line profiles, we constructed two-dimensional maps for the flux distributions, line ratios, radial velocities and gas velocity dispersions for the $\mathrm{H} \beta$ and [O III] emitting gas, similar to those of previous studies by our group (e.g., Barbosa et al. 2009). Continuum maps in the blue and red as well as the ratio between the two were also obtained. Extended emission is observed both in [O III] and $\mathrm{H} \beta$ with the [O III] flux distribution being more centrally concentrated, but extending to $1.6 \mathrm{kpc}$ from the nucleus to the north. The $\mathrm{H} \beta$ flux distribution is more extended than that of $[\mathrm{O} \mathrm{III}]$ along $\mathrm{PA} \sim 15^{\circ}$ and $195^{\circ}$, and is also observed in a detached patch from the nucleus, which seems to be associated with regions of recent star formation. Emission-line ratios support AGN ionization in the inner region $(0.3 \mathrm{kpc}$ from the nucleus) and a contribution from young stars in the surrounding regions. The gas velocity fields suggest the presence of rotation, with a possible contribution from outflows, notably in blueshift to the east, supported by an increase in the velocity dispersions at the same location. The continuum is redder at the nucleus than in the surrounding regions, indicating obscuration by dust or older stellar population. Principal component analysis (PCA) was conducted on the data cube, with the results suggesting that the AGN dominates the gas excitation in the inner $0.6 \mathrm{kpc}$ while recent star formation dominates in the surrounding regions. A compact rotation pattern of the gas around the nucleus is also reveiled by the PCA. More details about this study, as well as the corresponding figures will be presented in a forthcoming paper (Sanmartim et al. 2010, in preparation).

\section{References}

Allington-Smith, J., et al. 2002, PASP, 114, 892

Brotherton, Michael S., Stoll, R., Paul, C., Diamond-Stanic, A., Shang, Z., Cales, S., Ganguly, R., Canalizo, G., \& Vanden Berk, D. 2007, BAAS, 38, 95

Barbosa, F. K. B., Storchi-Bergmann, T., Cid Fernandes, R., Winge, C., \& Schmitt, H. 2009, MNRAS, 396, 2 